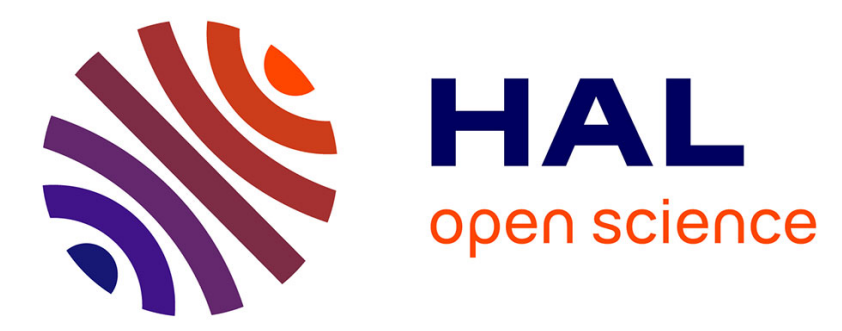

\title{
Atom interferometry as a detector of rotation and gravitational waves: comparison of various diffraction processes
}

Steven Lepoutre, Haikel Jelassi, Gérard Trénec, Matthias Büchner, Jacques

Vigué

\section{To cite this version:}

Steven Lepoutre, Haikel Jelassi, Gérard Trénec, Matthias Büchner, Jacques Vigué. Atom interferometry as a detector of rotation and gravitational waves: comparison of various diffraction processes. General Relativity and Gravitation, 2011, 43 (7), pp.2011-2025. 10.1007/s10714-010-1133-y . hal00534778

\section{HAL Id: hal-00534778 \\ https://hal.science/hal-00534778}

Submitted on 10 Nov 2010

HAL is a multi-disciplinary open access archive for the deposit and dissemination of scientific research documents, whether they are published or not. The documents may come from teaching and research institutions in France or abroad, or from public or private research centers.
L'archive ouverte pluridisciplinaire $\mathbf{H A L}$, est destinée au dépôt et à la diffusion de documents scientifiques de niveau recherche, publiés ou non, émanant des établissements d'enseignement et de recherche français ou étrangers, des laboratoires publics ou privés. 


\title{
Atom interferometry as a detector of rotation and gravitational waves: comparison of various diffraction processes
}

\author{
Steven Lepoutre • Haikel Jelassi • Gérard \\ Trénec • Matthias Büchner · Jacques Vigué
}

Received: date / Accepted: date

\begin{abstract}
In this paper, we discuss the optimization of atom interferometers for rotation and gravitational wave detection. We consider various diffraction processes and we focus our analysis on Bragg diffraction by a laser standing wave. We show that high order diffraction should be feasible with moderate laser power.
\end{abstract}

Keywords Atom interferometry · Bragg diffraction · Raman diffraction · Inertial sensor

PACS 03.75.Dg $\cdot 04.80 . \mathrm{Nn} \cdot 39.20 .+\mathrm{q}$

\section{Introduction}

Matter wave interferometers have a high sensitivity to inertial effects [1-4] as first illustrated by the observation of the effect of gravity on a neutron interferometer in 1975 [5]. Atom interferometers do not need a complex source as neutron interferometers and they are far more sensitive than neutron interferometers thanks to their considerably larger signal: several groups have thus obtained very impressive results quoted below. It is thus very natural to consider the use of atom interferometers as detectors of gravitational waves.

In an atom interferometer, there are two very important choices, namely the choice of the atom and the choice of the diffraction process. The atom used to build an atom interferometer is mostly chosen for practical reasons: the productions of an atomic beam or a cold sample must be easy; laser excitation and detection of the chosen atom must be feasible with convenient single frequency lasers.

Steven Lepoutre (1) · Haikel Jelassi (1,2) · Gérard Trénec (1) · Matthias Büchner (1) · Jacques Vigué (1)

(1) Laboratoire Collisions Agrégats Réactivité, IRSAMC, Université de Toulouse-UPS and CNRS UMR 5589, France

Tel.: $+33-561556016$

Fax: +33-61558317

E-mail: jacques.vigue@irsamc.ups-tlse.fr

(2) present address: CNSTN, Pôle Technologique. 2020 Sidi Thabet, Tunisia 
To build gyros, several experiments have used thermal atoms such as calcium [6], sodium [7] and caesium [8-10] but most atom interferometers devoted to inertial measurements have used cold atomic samples. Gyrometers have used caesium [11] or rubidium [12]; accelerometers devoted to the measurement of $g$ have used sodium [13,14], caesium $[15,16]$ or rubidium [17] and a test of the equivalence principle has been made with rubidium [18]; gravity gradiometers have used caesium $[19,20]$ or rubidium [21] and these gradiometers have been used to measure the Newtonian constant of gravity [21-23]. To save space, we will not quote here the inertial measurements done with Bloch oscillations.

In a very large fraction of these atom interferometers, the atom diffraction process is Raman diffraction first used in [13]. Several other diffraction processes are available: diffraction on material gratings first used by D. Pritchard and co-workers [24] and various types of laser diffraction processes as discussed by C. J. Bordé [4,25]. Among the other laser diffraction processes, we will focus here on Bragg diffraction by a laser standing wave, first observed by D. Pritchard and co-workers [26] and applied to atom interferometry by D. M. Giltner et al. [27].

These experiments have been extremely successful. As far as we know, the choice of the atom and the choice of the diffraction were made for practical reasons ant these two choices could not be the best ones. The goal of the present paper is to discuss these two choices from first principles.

\section{General considerations on atom interferometers}

An atom interferometer is formed of a very bright atom source (atomic beam, cold atom cloud released from a trap, atom laser emitted by a Bose-Einstein condensate) from which an atomic beam is extracted with a defined velocity and a high collimation. The simplest interferometer design is the Mach-Zehnder type and we will center the present discussion on this type of interferometer but other types of interferometers such as the Ramsey-Bordé interferometer are also frequently used and very interesting. In the Mach-Zehnder interferometer, the atomic beam is diffracted three times, in order to split, reflect and recombine the atomic wave, as illustrated in figure 1 . In the discussion, we will assume that the interferometer is a spatial interferometer: in such an interferometer, the laser standing waves are always present and the interaction time is fixed by the spatial width of these laser standing waves and the atom velocity. From the theoretical point of view, there are some subtle differences between spatial and temporal interferometers, in particular because the atom energy is strictly conserved in spatial interferometers and not in temporal ones. Although interesting from a fundamental point of view, these differences are not so important and we think that our discussion could be easily extended to the case of temporal interferometers. However, the physical arrangements for these two types of interferometers are quite different and we will consider here only the case of spatial interferometers. Figure 1 defines the axis used in the discussion of Bragg diffraction.

This type of interferometer produces two output beams with complementary signals given by:

$$
I_{i}=I_{0 i}\left[1+\mathcal{V}_{i} \cos \varphi\right]
$$

where $I_{0 i}$ is the mean intensity and $\mathcal{V}_{i}$ the fringe visibility of the output beam $i$ (if we use the same phase $\varphi$ for the two output beams, the visibility of one of the two 


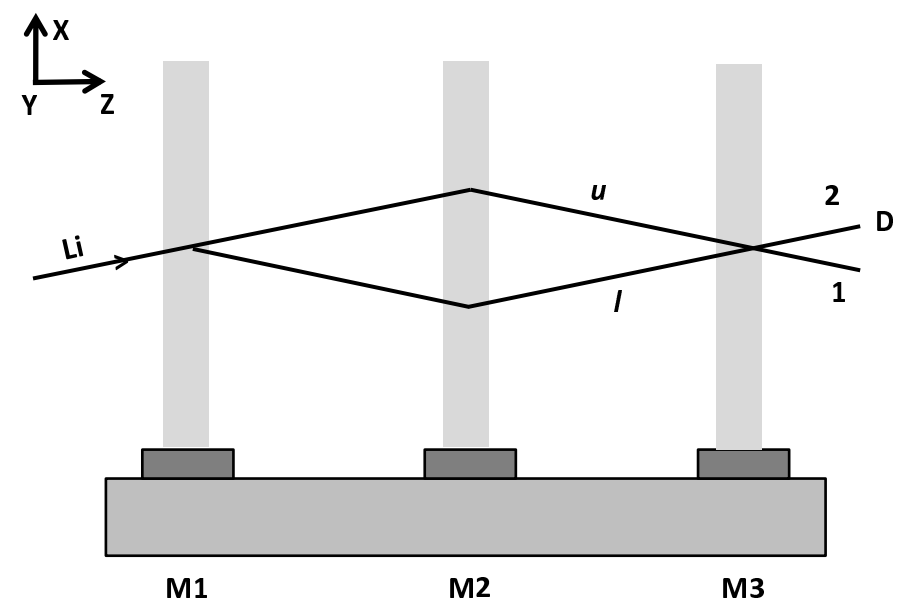

Fig. 1 Schematic drawing of a Mach-Zehnder atom interferometer using laser diffraction in the Bragg regime. The three laser standing waves are produced by reflecting the laser beams on the mirrors $M_{i}$. The thick lines represent the atomic beam paths and the output beams are labelled 1 and 2 . The axis used in the discussion are defined.

output beams is negative). The intensities are expressed as the number of detected particles per unit time. $\varphi$ is the phase of the fringe signal which can be swept by various techniques depending on the diffraction process. For an ideal interferometer (i.e. without any loss process), the complementary character of the output beams imposes that $I_{01} \mathcal{V}_{1}+I_{02} \mathcal{V}_{2}=0$.

The performance of an interferometer is measured by its phase sensitivity i.e. by the minimum phase-shift $\Delta \varphi_{\min }$ which can be detected in a unit time. Assuming that the noise is limited by Poisson noise, a reasonable assumption for ordinary atom sources, the minimum detectable phase-shift $\Delta \varphi_{\min }$ is given by:

$$
\Delta \varphi_{\min }=\frac{1}{\sqrt{I_{0 i}} \mathcal{V}_{i}}
$$

Obviously, to get the optimum phase sensitivity, the quantity $I_{0 i} \mathcal{V}_{i}^{2}$ must be maximized. The sensitivity to a rotation with an angular velocity $\Omega_{\text {rot }}$ is due to Sagnac effect and the associated phase-shift $\Delta \varphi_{\text {Sagnac }}$ is given by:

$$
\Delta \varphi_{\text {Sagnac }}=2 p k_{G} \Omega_{\text {rot }} v T^{2}
$$

where $p$ is the diffraction order, $k_{G}$ is the grating of wavevector $\left(k_{G}=2 \pi / a\right.$ where $a$ is the grating period), $v$ is the atom velocity and $T$ is the time between two consecutive diffraction events. Written in this form, the formula is valid for any type of wave (light wave or matter wave) and the large gain of sensitivity associated to matter waves appears if one introduces the distance $L=v T$, which is the size of the interferometer. The quantity $v T^{2}$ is equal to $L T=L^{2} / v$ and for a given size $L$, the sensitivity scales as $1 / v$. Finally, the Sagnac phase shift is proportional to the diffraction order $p$ and an interesting gain of sensitivity is expected if one can use a high value of $p$, with no degradation of the phase sensitivity. 
It is common to write the Sagnac phase shift as a function of the area $A_{a t}$. enclosed by an atom interferometer and one gets:

$$
\Delta \varphi_{\text {Sagnac,at. }}=2 \frac{m}{\hbar} A_{\text {at. }} \Omega_{\text {rot }}
$$

The sensitivity of a light interferometer operating with photons of energy $\hbar \omega$ is given by:

$$
\Delta \varphi_{\text {Sagnac }, \text { phot. }}=2 \frac{\omega}{c^{2}} A_{\text {phot. }} \Omega_{\text {rot }}
$$

Under the assumption of equal areas, the ratio of sensitivity is given by:

$$
\frac{\Delta \varphi_{\text {Sagnac }, \text { at }}}{\Delta \varphi_{\text {Sagnac,phot. }}}=\frac{m c^{2}}{\hbar \omega}
$$

which is very large, of the order of $10^{11}$ for a heavy atom and a $1-2 \mathrm{eV}$ photon. Even if the assumption of equal areas is not realistic, the very large sensitivity ratio predicted by equation 6 explains the interest for atomic interferometers for measurement of rotations.

The sensitivity to gravitational waves has been studied by many authors and we quote only some papers [28-33] but we will not reproduce the results of their calculations. In the simplest case, the phase shift is given by an equation similar to equation 4 , with $\Omega_{\text {rot }}$ replaced by an average of the perturbation of the metric tensor over the time spent by the atoms in the interferometer. The large gain of sensitivity associated to the use of an atom interferometer rather than a light interferometer predicted by equation 6 for the detection of rotation does not exist for the detection of gravitational waves. After some controversy, this point seems strongly established now. However, it remains interesting to estimate the parameters which optimize the sensitivity of atom interferometers for gravitational wave detection and this is equivalent to the optimization of the sensitivity to rotation given by equations 3 and 4 . As shown by the discussion following equation 3 , the use of slow atoms and of a large interferometer is necessary to maximize the quantity $L^{2} / v$ but this maximization will be practically limited on Earth by gravity.

We are going to discuss first the choice of the diffraction process and this study will be used to make the choice of the atom. We consider successively diffraction by a material grating and laser diffraction processes.

\section{Atom diffraction by material gratings}

Material gratings present the interest of having very small periods, as small as 100 $\mathrm{nm}$, and the gratings presently achieved are of high quality. These gratings produce several diffracted beams and their presence complicates the interferometer and reduces its transmission, which, for an optimized Mach-Zehnder interferometer, is calculated to be close to $1 \%$. Finally, because of the Van der Waals atom-surface interaction, the diffracted waves receive a phase shift which depends on the shape and dimensions of the grating wires, on the diffraction order and on the atom velocity [34,35]. When the atom velocity decreases, these phase shifts increase and the effective slit width diminishes [36]. 
Material gratings have been extensively used in a sodium atom interferometer with atom at thermal velocities near $1000 \mathrm{~m} / \mathrm{s}$ [24]. A helium atom Mach-Zehnder interferometer was operated by J. P. Toennies and co-workers, with lower velocities near 300 $\mathrm{m} / \mathrm{s}$. Because of the Van der Waals atom-surface interaction, we think that diffraction by material gratings cannot be easily used with very slow atoms, with velocities of the order a few $\mathrm{m} / \mathrm{s}$, and, as far as we know, no such interferometer has been operated.

\section{Common features of atom diffraction by laser}

Laser diffraction covers a wide variety of different processes, as discussed by C. J. Bordé $[4,25]$. Here we will discuss only two processes, namely Raman diffraction and Bragg elastic diffraction. In all cases, the elementary diffraction process is the absorption of a photon going in one direction followed by the stimulated emission of a photon going in the opposite direction. The corresponding grating period is equal to half the laser wavelength used for diffraction and, because the laser frequency must be chosen quasiresonant with the first resonance transition of the atom, the grating period is usually of the order of $300-400$ nanometers. This period is considerably larger than the period of material gratings but laser diffraction, which has many interesting properties, is very commonly used.

We are going to describe rapidly Raman diffraction, without going into details, because it has been used in many experiments, and we will focus more time on Bragg diffraction because it is less commonly used. Moreover, we think that Bragg diffraction at high diffraction orders is very promising but it is still under development as shown by recent important progresses [37,38].

\section{Raman diffraction}

Raman diffraction requires two single frequency laser contra-propagating beams with a phase-locked frequency difference equal to the ground state hyperfine structure splitting. The diffraction process is illustrated by figures 2 and 3. The laser frequency must be chosen very close to one component of the first resonance transition of the atom, with a frequency detuning $\delta$ usually chosen of the order of a few GHz. Such a small value of $\delta$ is necessary to keep the laser power density to an accessible value but a non-zero detuning is absolutely needed to reduce the probability of photon absorption followed by a spontaneous emission process which destroys the coherence of the atom wave.

Raman diffraction presents a great advantage: the direct and diffracted beams are in two different internal states and a state selective detection measures separately the intensity carried by the two output beams of an atom interferometer. Raman diffraction naturally operates only at first order: it is impossible to do $p$ absorption-stimulated emission because, after one cycle, the atom has changed its internal state and this change cannot be repeated. A trick to circumvent this difficulty is to interchange the direction of the two laser beams of frequencies $\omega_{1}$ and $\omega_{2}$ as done by Weiss and coworkers for the measurement of $\hbar / m_{C s}[39]$. 


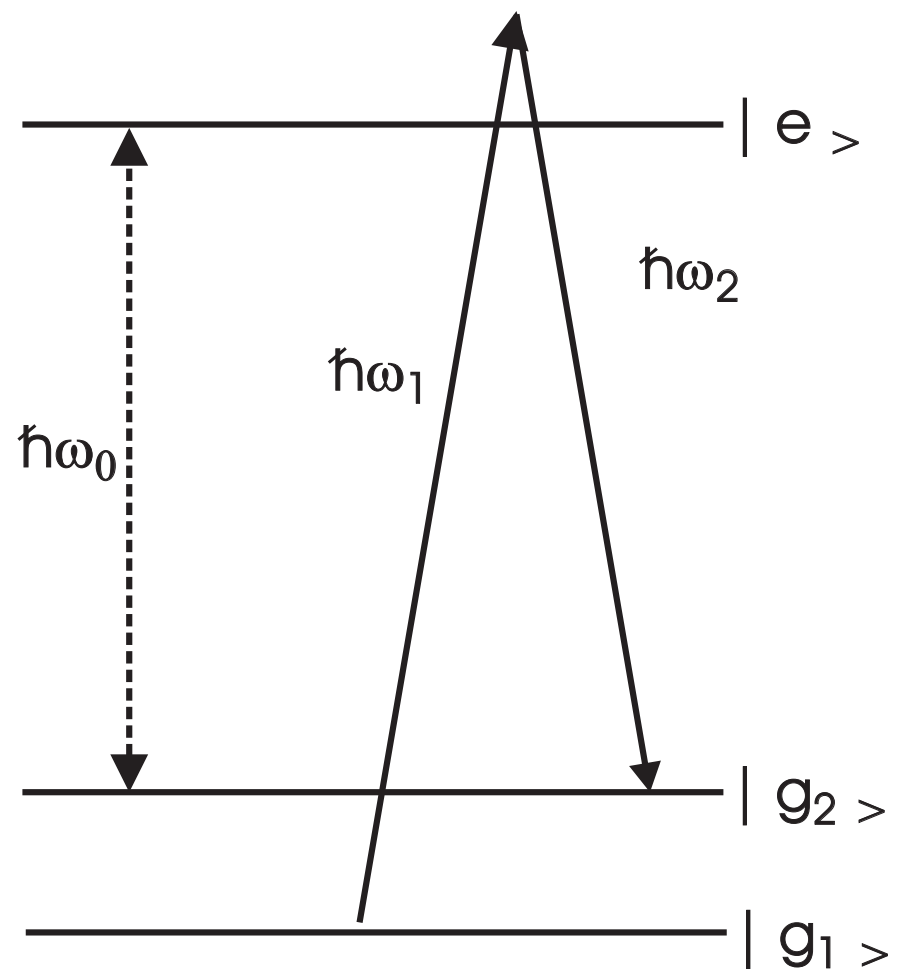

Fig. 2 Principle of atom diffraction by laser using a Raman process: the atom absorbs a photon of energy $\hbar \omega_{1}$ and emits a photon of energy $\hbar \omega_{2}$ by stimulated emission, thus making a transition from state $\left|g_{1}\right\rangle$ to state $\left|g_{2}\right\rangle$. The case of elastic diffraction is deduced from the Raman case by making $\left|g_{1}\right\rangle=\left|g_{2}\right\rangle$ and $\hbar \omega_{1}=\hbar \omega_{2}$. In this last case, diffraction of order $p$ can be observed, as the absorption-stimulated emission cycle may occur $p$ times.

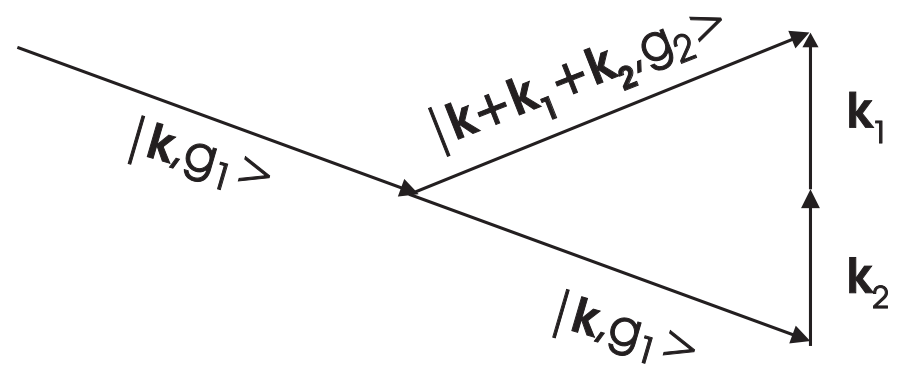

Fig. 3 Momentum conservation in atom diffraction by laser: the atom receives a momentum kick equal to $\hbar \mathbf{k}_{1}$ due to the absorption of a photon of energy $\hbar \omega_{1}$ going in one direction and a second kick equal to $\hbar \mathbf{k}_{2}$ in the same direction, due to the stimulated direction of a photon of energy $\hbar \omega_{2}$ going in the opposite direction. The conservation of the atom kinetic energy requires the Bragg geometry, as illustrated in this figure. As in Fig. 2, elastic diffraction is deduced from the Raman case by making $\hbar \mathbf{k}_{1}=\hbar \mathbf{k}_{2}$. 


\section{Bragg diffraction on a laser standing wave}

\subsection{General features}

The process involves a standing wave, produced by a single frequency laser and it is very similar to Raman diffraction illustrated by figures 2 and 3 but, now, the two photons have the same frequency and the atom initial and final states are identical, exactly as for diffraction by a material grating. Because of this last property, the direct and diffracted beams are in the same internal state. This property is interesting for atom interferometers, as in the two interferometer arms, the atom is in the same internal state and an homogeneous magnetic or electric stray field does not create any phase shift and a field gradient is necessary to create such a phase shift. In interferometers using Raman diffraction, because the atom is not in the same internal state in both arms, an homogeneous stray field is sufficient to create a phase shift of the fringe signal and a better control of the stray fields is needed. However, for an atom interferometer based on Bragg diffraction, the two output beams can be distinguished only by their momenta. This is feasible only if the projection of the initial momentum on the laser propagation axis is well defined, with a distribution width smaller than the recoil momentum $\hbar k_{L}$ (where $k_{l}$ is the laser wave vector). If this condition is fulfilled, Bragg diffraction can be very selective if the interaction time with the laser standing wave is long enough and only two diffracted beams are produced, corresponding to the order 0 (direct transmitted beam) and to the order $p$. Shorter interaction times can be also used (Raman-Nath regime) with several diffraction orders being produced. As a very narrow momentum distribution is not easy to achieve, the use of Bragg diffraction with cold atoms has been restricted for a long period to experiments either with thermal atoms (see below) or with Bose-Einstein condensates (for instance $[40,41]$ ).

A very interesting feature of Bragg diffraction is that it is possible to use high values of the diffraction order $p$ in order to increase simultaneously the interferometer area $A_{a t}$. and the inertial phase shifts. This order is chosen by adjusting the projection of the initial momentum $\hbar \mathbf{k}_{i}$ on the laser propagation axis taken as the $x$ axis so $\hbar k_{i x}= \pm p \hbar k_{L}$. The highest diffraction order achieved with Bragg diffraction has steadily increased. After the observation of first order diffraction by P. J. Martin et al. in 1988 [26], diffraction of order $p=6$ was observed by Giltner et al. in 1995 [42], of order $p=8$ by A. E. A. Koolen et al. in 2002 [43] and of order $p=12$ by H. Müller et al. [38] in 2008.

The first atom interferometers using Bragg diffraction were Mach-Zehnder interferometers operated with thermal atoms: they were built in 1995 by D. M. Giltner et al. [27], using metastable neon and laser diffraction of order $p=1,2$ and 3, and by E. M. Rasel et al. [44] using metastable argon and laser diffraction of order $p=1$ (in this case, diffraction was in the Raman-Nath regime). In 2001, our group has built a lithium atom similar interferometer $[45,46]$, using laser Bragg diffraction of order $p=1,2$ and 3 .

With ultra-cold atoms, the first interferometer was built by Torii et al. [41] in 2000: it was a Mach-Zehnder interferometer using a free falling rubidium Bose-Einstein. In 2005, Y. J. Wang et al. [47] have built an atom Michelson interferometer on a chip using a Bose-Einstein condensate and Raman-Nath diffraction. In 2006, O. Garcia et al. [48] have built a Michelson interferometer using a rubidium Bose-Einstein condensate as atom source and, in 2008, H. Müller et al. [38] have built Mach-Zehnder and RamseyBordé interferometers using cold cesium atoms and diffraction orders up to $p=12$. 
We may remark that, for both diffraction processes (Raman and Bragg), a narrow distribution of the projection $\hbar k_{i x}$ of the initial momentum on the laser propagation axis is also needed to insure a constant diffraction amplitude. Diffraction can be described as a Rabi oscillation between two motional states of the atom [50] and this condition results from the fact that diffraction can reach a large probability only if the Rabi oscillation is resonant. For the atoms with $\hbar k_{i x}$ differing noticeably from $\pm p \hbar k_{L}$, the diffraction amplitude differs strongly from the one for resonant atoms. In the case of Raman diffraction, a broad distribution of $\hbar k_{i x}$ will dilute the atoms which are diffracted in a large number of atoms for which the diffraction probability is low and this dilution reduces at the same time the fringe visibility $\mathcal{V}$ and, following equation 2 , the interferometer phase sensitivity.

\subsection{Theory of Bragg diffraction at high order}

There is a large amount of papers concerning diffraction by a laser standing wave and we refer the reader to three papers. A paper by C. Keller et al. [49] in 1999 and a paper of our group [50] in 2001 have introduced the two main dimensionless parameters characterizing this diffraction process. More recently, H. Müller et al. [37] have discussed Bragg diffraction at high orders and the non-adiabatic effects due to the envelope of the laser pulse. In our paper [50], we considered square laser pulses which lead to analytic results but they are not realistic, in particular for spatial interferometers, and not favorable for a selective Bragg diffraction. We will use the results of H. Müller et al. [37] for Gaussian pulses but let us first recall the two dimensionless parameters for atom diffraction by a laser standing wave $[49,50]$ :

- for a square pulse of laser light of duration $t_{i n t}$, the dimensionless duration is $\tau_{\text {int }}=\omega_{\text {rec }} t_{i n t}$, with $\omega_{\text {rec }}=\hbar k_{L}^{2} /(2 m)$ being the atom recoil frequency.

- the laser standing wave creates a potential $V(x)=V_{\max } \cos ^{2}\left(k_{L} x\right)$ discussed below. To measure the strength of this potential, a dimensionless parameter $q=$ $V_{\max } /\left(4 \hbar \omega_{r e c}\right)$ was introduced. The quantum motion along the direction $x$ is described by Mathieu's equation (chapter 20 of ref. [51]) and the present definition of $q$ coincides with the definition of the traditional $q$ parameter of Mathieu's equation.

- H. Müller et al. [37] do not use the same notations but they also use the recoil frequency $\omega_{\text {rec }}$ to scale the potential strength and the pulse duration.

In order to make the calculation clear, let us start from the beginning. First, we refer the reader to the book Atom-photon interactions by C. Cohen-Tannoudji et al. [54]. The electric field of the laser standing wave is given by:

$$
\begin{aligned}
E & =E_{0}(y, z)\left[\cos \left(\omega_{L} t+k_{L} x\right)+\cos \left(\omega_{L} t-k_{L} x\right)\right] \\
& =2 E_{0}(y, z) \cos \left(\omega_{L} t\right) \cos \left(k_{L} x\right)
\end{aligned}
$$

where $E_{0}$ is the electric field of one of the incident laser beam. To simplify the equations, the polarization effects are omitted. It is important to relate the electric field to the laser beam parameters. We assume a Gaussian beam, close to its waist, with a transverse field dependence given by:

$$
E_{0}(y, z)=E_{m} \exp \left[-\frac{y^{2}}{w_{y}^{2}}-\frac{z^{2}}{w_{z}^{2}}\right]
$$


We have considered that the laser beam is characterized by different waist radius in the $y$ and $z$ directions: a smaller value of the $w_{z}$ radius increases the electric field $E_{m}$ while a large value of the $w_{y}$ radius is necessary to use a larger height of the atomic beam in order to enhance the signal. The maximum electric field $E_{m}$ is related to the power $P$ transported by the incident laser beam by:

$$
E_{m}^{2}=4 \mu_{0} c \frac{P}{\pi w_{y} w_{z}}
$$

The atom-laser coupling is described by the Rabi frequency $\Omega_{1}$ given by:

$$
\hbar \Omega_{1}=-2 d_{e g} E_{0}(y, z) \cos \left(k_{L} x\right)
$$

where $d_{e g}$ is the transition dipole matrix element for the $e-g$ transition, assumed to be real. If the laser frequency detuning $\delta=\omega_{L}-\omega$ is sufficiently large and the electric field not too large, the atom remains mostly in its ground state where it feels a potential $V$ given by:

$$
V=\frac{\left(\hbar \Omega_{1}\right)^{2}}{4 \hbar \delta}=\frac{d_{e g}^{2} E_{0}^{2}(y, z)}{\hbar \delta} \cos ^{2}\left(k_{L} x\right)
$$

However, the atom, which has a weak probability of being excited, emits fluorescence photons with a rate:

$$
\begin{aligned}
\frac{d N}{d t} & =\frac{\gamma}{2} \times \frac{\Omega_{1}^{2}}{2 \delta^{2}} \\
& =\gamma \frac{d_{e g}^{2} E_{0}^{2}(y, z) \cos ^{2}\left(k_{L} x\right)}{\hbar^{2} \delta^{2}} \\
& =\gamma \frac{d_{e g}^{2} E_{0}^{2}(y, z)}{2 \hbar^{2} \delta^{2}}
\end{aligned}
$$

In the last line, we have replaced the $\cos ^{2}\left(k_{L} x\right)$ term by its average $1 / 2$. When an atom of velocity $v$ crosses a laser standing wave with the profile given by equation 8 , the total probability of spontaneous emission is given by:

$$
P_{s p . e .}=\int \frac{d N}{d t} \frac{d z}{v}=\sqrt{\frac{\pi}{2}} \times \frac{\gamma w_{z}}{v} \times \frac{d_{e g}^{2} E_{m}^{2}}{2 \hbar^{2} \delta^{2}}
$$

where we have assumed that $y=0$. It is important to keep $P_{s p . e .} \ll 1$ and this condition will fix the minimum value of the detuning $\delta$.

H. Müller et al. [37] have discussed the non-adiabatic effects assuming Gaussian laser pulses and, from now on, we will follow their notations: their $\Omega \cos ^{2}\left(k_{L} x\right)$ (we have transformed their $z$ axis into our $x$ axis) is equal to our $V / 2 \hbar$ and we may identify their pulse (their equation 60):

$$
\Omega=\bar{\Omega} \exp \left[-\frac{t^{2}}{2 \sigma^{2}}\right]
$$

to our equation 11, using the electric field given by equation 8 and assuming an atom velocity $v$ (i.e. $z=v t$ ) and maximum interaction (i.e. $y=0$ ). By identification, we get $\bar{\Omega}=d_{e g}^{2} E_{m}^{2} /\left(2 \hbar^{2} \delta\right)$ and $\sigma=w_{z} /(2 v)$. 
When using diffraction of order $p$, the Rabi oscillation of the atom couples two motional states with opposite momenta $\hbar k_{x}= \pm p \hbar k_{L}$. In perturbation theory, the first non vanishing order term coupling these states appears at order $p$ and is given by $\Omega_{\text {eff }}=\Omega^{p} /\left[\left(8 \omega_{r e c}\right)^{p-1}[(p-1) !]^{2}\right]$ as first demonstrated by D. M. Giltner [42]. This result suggests that high order diffraction will require very large laser power densities because of the dependence in $\Omega^{p}$ and of the small prefactor but this is not true because, in the range of parameters of practical importance, this leading term is not sufficient and higher order terms must be taken into account. Moreover, this coupling term is equal to the difference $\left(a_{p}-b_{p}\right)$ where these quantities are defined for Mathieu's equation [51] and the expansions of $a_{p}$ and $b_{p}$ in powers of $q$ have a finite radius of convergence $\rho$. For $a_{0}$, this radius is $q=1.468$ (see reference [51] and references therein). For higher $p$ values, the radius of convergence of $a_{2 n}, a_{2 n+1}, b_{2 n}$ and $b_{2 n+1}$ have been calculated up to $n=9$. The radius of convergence of the expansions of these quantities in powers of $q$ scale with $n$ like $2.042 n^{2}$ for the dominant in $n$ term [52]. The asymptotic behavior when $n$ goes to infinity of this radius of convergence $\rho$ by H. Volkmer [53] is shown to verify $\rho>2.0418 n^{2}$. Power expansions for $a_{p}$ and $b_{p}$ are known [55] and can be calculated by the Mathieu package of Maple software; when $q$ is equal to a few unity, these expansions appear to be slowly convergent. Obviously, the power expansion of the difference $\left(a_{p}-b_{p}\right)$ might be convergent even when the expansions of $a_{p}$ and $b_{p}$ are divergent.

As discussed by H. Müller et al. [37], a low-loss Bragg diffraction process is possible, even at a high order $p$, if the laser pulse used for Bragg diffraction has a width parameter $\sigma$ such that:

$$
\omega_{r e c} \sigma>A \frac{p^{1 / 6}}{p-1}
$$

with a prefactor $A$ depending on how large losses are accepted ( $A=0.38$ for losses below $10^{-2}$ and $A=1.5$ for losses below $10^{-10}$ ). This result is counterintuitive and very important as it proves that high order diffraction can be done with an interaction time which decreases with increasing order $p$ and that, for a given order, the interaction time scales like $1 / \omega_{\text {rec }}$.

In figure 4 of their paper, H. Müller et al. [37] have plotted the parameters of a Gaussian pulse corresponding to a $\pi$ diffraction pulse as a function of the order $p$ from 4 to 9 . We have measured on this figure the values of $\bar{\Omega}_{\pi}(p) / \omega_{\text {rec }}$ with a pulse width $\sigma$ such that $\omega_{r e c} \sigma=p^{1 / 6} /(p-1)$ which corresponds to a low-loss diffraction process. In this range, $4 \leq p \leq 9$, the values $\bar{\Omega}_{\pi}(p) / \omega_{\text {rec }}$ are well approximated by $\bar{\Omega}_{\pi} / \omega_{\text {rec }} \approx 20(p-3)$ (the plot presents a weak upward curvature which can be neglected here). This result proves that high-order diffraction is feasible with a laser power density which increases more or less linearly with the order $p$. The product $\bar{\Omega} \sigma$ of the potential strength $\bar{\Omega}$ by the interaction time scale $\sigma$ is a slowly varying function of the diffraction order $p$ given by:

$$
\bar{\Omega} \sigma \approx A \frac{20(p-3) p^{1 / 6}}{p-1}
$$

in the range $4 \leq p \leq 9$. The $\bar{\Omega} \sigma$ is given by:

$$
\bar{\Omega} \sigma=\frac{d_{e g}^{2}}{\hbar \delta} \times \frac{\mu_{0} c}{\pi} \times \frac{P}{w_{y}}
$$


It is important to note that $w_{z}$ has disappeared from this last equation and this explains why the needed laser power $P$ increases slowly with the diffraction order $p$.

Let us now discuss the choice of the detuning $\delta$. A small $\delta$ value increases at the same time the potential strength, like $1 / \delta$, and the probability of spontaneous emission,

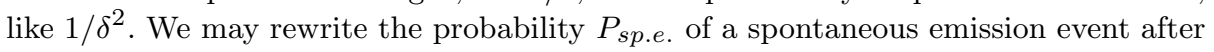
crossing a laser standing wave:

$$
P_{\text {sp.e. }}=\frac{\gamma}{\delta} \bar{\Omega} \sqrt{\frac{\pi}{2}} \times \frac{w_{z}}{v}
$$

If we combine this result with the value of $\sigma=w_{z} /(2 v)$, we get :

$$
\bar{\Omega} \sigma=\frac{\delta}{\gamma \sqrt{2 \pi}} P_{s p . e .}
$$

For a fixed value of the spontaneous emission probability $P_{s p . e .}$, the product $\bar{\Omega} \sigma$ depends only on the ratio $\delta / \gamma$ and is independent of the other atom properties.

\section{Choice of the atom}

We will consider here only three alkali atoms, namely lithium, rubidium and caesium. We will not consider any metastable atom, such as helium and rare gases, because the available beam intensities are considerably smaller than with ground state atoms.

Because of the equivalence principle, the atom mass is absent from the equations giving the sensitivity to acceleration or rotation and also to gravitational waves but the atom mass plays an important role in the diffraction process through its recoil frequency $\omega_{r e c}=\hbar k_{L}^{2} /(2 m)$. The other important parameters are all linked to the resonance transition used to diffract:

- the laser wavelength $\lambda$ used to diffract the atoms defines the grating period $k_{G}=$ $4 \pi / \lambda$ which is important in the interferometer sensitivity.

- the atom recoil frequency $\omega_{r e c}$ defines the time scale of the diffraction process.

- the resonance state natural width $\gamma$ fixes the minimum value of the detuning needed to insure a coherent diffraction process i.e. with a negligible spontaneous emission probability.

- the resonance transition dipole moment $d_{e g}$ which is necessary to calculate the potential ( $d_{e g}, \lambda$ and $\gamma$ are well known to be related).

Table 1 Main parameters for laser diffraction of lithium, rubidium and caesium: wavelength $\lambda$ of the $\mathrm{D}_{2}$ line; reduced matrix element of dipole moment of the ${ }^{2} S-{ }^{2} P_{3 / 2}$ transition; decay

\begin{tabular}{|c|c|c|c|}
\hline Atom & lithium 7 & rubidium 87 & caesium 133 \\
\hline$\lambda(\mathrm{nm})$ & 671 & 780 & 852 \\
\hline$<g\|d\| e>\left(10^{-29}\right.$ C.m $)$ & 3.98 & 3.58 & 3.80 \\
\hline$\gamma\left(10^{7} \mathrm{~s}^{-1}\right)$ & 3.69 & 3.81 & 3.29 \\
\hline$\omega_{r e c}\left(\mathrm{~s}^{-1}\right)$ & $3.965 \times 10^{5}$ & $2.369 \times 10^{4}$ & $1.298 \times 10^{4}$ \\
\hline
\end{tabular}
rate $\gamma$ of the ${ }^{2} P_{3 / 2}$ state and recoil frequency $\omega_{\text {rec }}$. 
We consider that the laser is quasi-resonant with the first resonance transition because, in the alkali atoms, the oscillator strength of the transitions connecting the ${ }^{2} S$ ground to upper ${ }^{2} P$ states are considerably weaker than for the first resonance line. Moreover, the wavelength of the first resonance line of the alkali atoms is in a very convenient spectral region, where powerful continuous single frequency lasers are available. We have collected in table 1 the value of these parameters for lithium, rubidium and caesium

This table proves clearly that the only parameter which varies much among these three alkali atoms is the recoil frequency $\omega_{r e c}$. Equation 15 gives the minimum acceptable value of the product of the laser duration parameter $\sigma$ by the recoil frequency $\omega_{r e c}$. Clearly, if one wants to use a high order diffraction and a short diffraction time, the best choice is the atom with the largest recoil frequency $\omega_{r}$ and lithium will be the best choice. Bragg diffraction of a light atom can be made in a considerably shorter time than with an heavy atom.

However, we have calculated the condition (equation 15) verified by the pulse length $\sigma$ in the case of lithium, with $A=1$ and $p=9$, and we find $\sigma>5 \times 10^{-7} \mathrm{~s}$. This lower limit is not easy to approach in a spatial interferometers with slow atoms $(v \approx 10 \mathrm{~m} / \mathrm{s})$, because it corresponds a very small waist $w_{z} \approx 5 \times 10^{-6} \mathrm{~m}$. Larger $\sigma$ and $w_{z}$ values can obviously be used but this may increase the needed laser power. A numerical model of the diffraction process will be necessary for a full optimization of a high-order Bragg Mach-Zehnder atom interferometer.

\section{Conclusion}

In this paper, we have compared the three diffraction processes which have been used to build atom interferometers. We have focussed our discussion on Bragg diffraction by a laser standing waves because this diffraction process can be used at high diffraction orders with a good selectivity.

We have shown that the laser power density needed for Bragg diffraction at large orders scales roughly like the order $p$. As the minimum interaction time needed to preserve Bragg selectivity scales roughly like $1 / p$, we predict that the laser power needed has only a slow dependence with the diffraction order $p$. In the same manner, the laser power density scales with the atom recoil frequency $\omega_{r e c}$ but the interaction time scales like $1 / \omega_{\text {rec }}$ and we thus predict that the necessary laser power has no direct dependence with the atom recoil frequency $\omega_{r e c}$. This means that Bragg diffraction at high order, which has been recently demonstrated with caesium by H. Müller et al. [38], should also be feasible with lighter atoms, including lithium 7 for which the recoil frequency is 30.6 times larger than for caesium.

High order Bragg diffraction is not the only way of increasing the separation of the paths in atom interferometers and Large Momentum Transfer (LMT) beam splitters have been developed, based on the principle of Bloch oscillations. In 2002, J. Hecker Denschlag et al. [56] have thus demonstrated splittings of a Bose-Einstein condensate with a momentum transfer up to $12 \hbar k$. Very recently, two interferometers using this type of LMT beam splitters have been operated by P. Cladé et al. [57] and by $\mathrm{H}$. Müller et al. [58]. These recent results, which may reveal very important to increase the sensitivity of atom interferometry for rotation and gravitational wave measurements, have not been discussed here. 
An important practical problem for the development of very high sensitivity inertial sensors comes from the fact that the laboratory frame is not an inertial frame: vibrations of the laboratory, which are detected by the interferometers, induce an important phase noise and loss of fringe visibility [10,59]. Fortunately, this vibration induced phase noise can be reduced by using an active suspension. Moreover, the rotation of the Earth induces a large Sagnac phase shift which depends on the atom velocity and the dispersion of this phase shift associated to the atom velocity distribution will also induce a loss of fringe visibility.

Acknowledgements We thank CNRS department MPPU, Région Midi-Pyrénées, ANR (grant ANR-05-BLAN-0094) and IRSAMC for support. We thank H. Volkmer for helpful correspondence.

\section{References}

1. A. W. Overhauser and R. Colella, Phys. Rev. Lett. 33, 1237 (1974)

2. J. Anandan, Phys. Rev. D 15, 1448 (1977)

3. J. F. Clauser, Physica B 151, $262(1988)$

4. C. J. Bordé, Phys. Lett. A 140, 10 (1989)

5. R. Colella, A. W. Overhauser, S. A. Werner, Phys. Rev. Lett. 34, 1472 (1975)

6. F. Riehle, Th. Kisters, A. Witte, J. Helmcke and Ch. J. Bordé, Phys. Rev. Lett. 67, 177 (1991)

7. A. Lenef, T. D. Hammond, E. T. Smith, M. S. Chapman, R. A. Rubenstein and D. E. Pritchard, Phys. Rev. Lett. 78, 760 (1997)

8. T. L. Gustavson, P. Bouyer and M. A. Kasevich, Phys. Rev. Lett. 78, 2046 (1997)

9. T. L. Gustavson, A. Landragin and M. A. Kasevich, Class. Quantum Grav. 17, 2385 (2000)

10. D. S. Durfee, Y. K. Shaham and M. A. Kasevich, Phys. Rev. Lett. 97, 240801 (2006)

11. B. Canuel, F. Leduc, D. Holleville, A. Gauguet, J. Fils, A. Virdis, A. Clairon, N. Dimarcq,

Ch. J. Bordé, A. Landragin and P. Bouyer, Phys. Rev. Lett. 97, 010402 (2006)

12. T. Müller, M. Gilowski, M. Zaiser, P. Berg, Ch. Schubert, T. Wendrich, W. Ertmer, and E. M. Rasel,Eur. Phys. J. D 53, 273281 (2009)

13. M. Kasevich and S. Chu, Phys. Rev. Lett. 67, 181 (1991)

14. M. Kasevich and S. Chu, Appl. Phys. B 54, 321 (1992)

15. A. Peters, K. Y. Chung and S. Chu, Nature 400, 849 (1999)

16. A. Peters, K. Y. Chung and S. Chu, Metrologia 38, 25 (2001)

17. J. Le Gouët, T.E. Mehlstäubler, J. Kim, S. Merlet, A. Clairon, A. Landragin and F. Pereira Dos Santos, Appl. Phys. B 92, 133144 (2008)

18. S. Fray, C. Alvarez Diez, T. W. Hänsch and M. Weitz, Phys. Rev. Lett. 93, 240404 (2004)

19. M. J. Snadden, J. M. McGuirk, P. Bouyer, K. G. Haritos and M. A. Kasevich, Phys. Rev. Lett. 81, 971 (1998)

20. J. M. McGuirk, G. T. Foster, J. B. Fixler, M. J. Snadden and M. A. Kasevich, Phys. Rev. A 65, $033608(2002)$

21. A. Bertoldi, G. Lamporesi, L. Cacciapuoti, M. de Angelis, M. Fattori, T. Petelski, A. Peters, M. Prevedelli, J. Stuhler and G.M. Tino, Eur. Phys. J. D 40, 271 (2006)

22. J. B. Fixler, G. T. Foster, J. M. McGuirk and M. A. Kasevich, Science 315, 74 (2007)

23. G. Lamporesi, A. Bertoldi, L. Cacciapuoti, M. Prevedelli and G. M. Tino, Phys. Rev. Lett. 100, $050801(2008)$

24. D. W. Keith, C. R. Ekstrom, Q. A. Turchette and D. E. Pritchard, Phys. Rev. Lett. 66, 2693 (1991)

25. C. J. Bordé, Atom interferometry, edited by P. R. Berman, (Academic Press, 1997) p. 257 26. P. J. Martin, B. G. Oldaker, A. H. Miklich and D. E. Pritchard, Phys. Rev. Lett., 60, 515 (1988)

27. D.M. Giltner, R. W. McGowan and Siu Au Lee, Phys. Rev. Lett. 75, 2638 (1995)

28. B. Lamine, M.-T. Jaekel and S. Reynaud, Eur. Phys. J. D 20, 165 (2002)

29. S. Reynaud et al., Int. J. Mod. Phys. A 17, 1003 (2002)

30. B. Lamine, Thèse Université Pierre et Marie Curie (2004), http://tel.archivesouvertes.fr/docs/00/04/71/24/PDF/tel-00006936.pdf 
31. A. Roura et al., Phys. Rev. D 73, 084018 (2006)

32. G. M. Tino and F. Vetrano, Class. Quantum Grav. 24, 2167 (2007)

33. S. Dimopoulos et al., Phys. Rev. Lett. 98, 111102 (2007)

34. J. D. Perreault and A. D. Cronin, Phys. Rev. Lett. 95, 133201 (2005)

35. S. Lepoutre, H. Jelassi, V.P.A. Lonij, G. Trénec, M. Büchner, A. D. Cronin and J. Vigué, Europhys. Lett. 88, 2002 (2009)

36. R. E. Grisenti, W. Schöllkopf, J. P. Toennies, G. C. Hegerfeldt and T. Köhler, Phys. Rev. Lett. 83, 1755 (1999)

37. H. Müller, S.-W. Chiow and S. Chu, Phys. Rev A 77023609 (2008)

38. H. Müller, S.-W. Chiow, Q. Long, S. Herrmann and S. Chu, Phys. Rev Lett. 100, 180405 (2008)

39. D. S. Weiss, B. C. Young and S. Chu, Appl. Phys. B 59, 217 (1994)

40. L. Deng, E. W. Hagley, J.Wen, M. Trippenbach, Y. Band, P. S. Julienne, J. E. Simsarian, K. Helmerson, S. L. Rolston and W. D. Phillips, Nature 398, 218 (1999)

41. Y. Torii Y. Suzuki, M. Kozuma, T. Sugiura, T. Kuga, L. Deng and E. W. Hagley, Phys. Rev. A, 61, 041602 (2000)

42. D.M. Giltner, R. W. McGowan and Siu Au Lee, Phys. Rev. A 52, 3966 (1995)

43. A. E. A. Koolen, G. T. Jansen, K. F. E. M. Domen, H. C. W. Beijerinck and K. A. H. van Leeuwen, Phys. Rev. A 65, 041601(R) (2002)

44. E. M. Rasel, M. K. Oberthaler, H. Batelaan, J. Schmiedmayer and A. Zeilinger, Phys. Rev. Lett. 75, 2633 (1995)

45. R. Delhuille, C. Champenois, M. Büchner, L. Jozefowski, C. Rizzo, G. Trénec and J. Vigué, Appl. Phys. B 74, 489 (2002)

46. A. Miffre, M. Jacquey, M. Büchner, G. Trénec and J. Vigué, Eur. Phys. J. D 33, 99 (2005) 47. Y.-J. Wang, D. Z. Anderson, V. M. Bright, E. A. Cornell, Q. Diot, T. Kishimoto, M. Prentiss, R. A. Saravanan, S. R. Segal and S. Wu, Phys. Rev Lett. 94, 090405 (2005)

48. O. Garcia et al., Phys. Rev. A, 74, 031601 (2006)

49. C. Keller, J. Schmiedmayer, A. Zeilinger, T. Nonn, S. Dürr, G. Rempe, Appl. Phys. B 69, 303 (1999)

50. C. Champenois, M. Büchner, R. Delhuille, R. Mathevet, C. Robilliard, C. Rizzo and J. Vigué, Eur. Phys. J. D 13, 271 (2001)

51. M. Abramowitz and I. A. Stegun, Handbook of Mathematical Functions (Dover, New York, 1965).

52. J. Meixner, F. W. Schäfke and G. Wolf, Lectures Notes In Mathematics 837, edited by A. Dold and B. Eckmann, Springer (1980)

53. H. Volkmer, Math. Nachr. 192, 239 (1998)

54. C. Cohen-Tannoudji, J. Dupont-Roc, G. Grynberg, Atom-photon interactions, Wiley Science Paperback (1998)

55. D. Frenkel and R. Portugal, J. Phys. A. 34, 3541 (2001)

56. J. Hecker Denschlag, J. E. Simsarian, H. Häffner, C. McKenzie, A. Browaeys, D. Cho, K. Helmerson, S. L. Rolston and W. D. Phillips, J. Phys. B: At. Mol. Opt. Phys. 35, 3095 (2002)

57. P. Cladé, S. Guellati-Khélifa, F. Nez, and F. Biraben, Phys. Rev Lett. 102, 240402 (2009)

58. H. Müller, S.-W. Chiow, S. Herrmann and S. Chu, Phys. Rev Lett. 102, 240403 (2009)

59. A. Miffre, M. Jacquey, M. Büchner, G. Trénec and J. Vigué, Appl Phys. B 84, 617 (2006) 